\title{
TIPOLOGÍA DE LOS ERRORES EN EL CÁLCULO MENTAL. UN ESTUDIO EN EL CONTEXTO EDUCATIVO
}

\author{
GóMEZ ALFONSO, B. \\ Departamento de Didáctica de la Matemática. Universitat de València.
}

\section{SUMMARY}

The present paper deals with a typology of errors and difficulty of Spanish students in a Teacher Training College when they apply historical mental computation methods (methods taken from the written tradition found in arithmetic textbooks) to solve problems with natural numbers and with decimal numbers.

\section{PRESENTACIÓN}

El informe que aquí se presenta resume un trabajo de tesis doctoral (Gómez, 1994) realizado sobre un tema del currículo del período de la educación obligatoria en España y en otros países. Este tema es el cálculo mental.

La investigación constó de dos partes ptincipales: una panorámica y otra experimental, de las cuales se podría decir que la primera se orientó hacia el contenido, más concretamente, hacia lo que es el saber transmitido por la tradición escrita, y la segurda, hacia su adquisición, es decir, hacia la elaboración de una propuesta de enseñanza de ese saber y el posterior estudio de la forma en que los estudiantes se lo apropian y utilizan.

\section{Precisando:}

1. El estudio panorámico se enfocó hacia el análisis de cómo se han configurado los métodos variados de cálcuto a lo largo de su historia como objetivo educativo, a la vista de tal y como han quedado reflejados en las aritméticas antiguas y modernas y en los documentos oficiales que determinan el currículo general y público español.

Asimismo, se buscó elaborar un catálogo, compendio y síntesis de los métodos de cálculo mental, actualizados en su lenguaje y organizados en torno a una estructura global y unificadora para las cuatro operaciones.
Esta estructura se constituyó en una parte importante de la propuesta experimental que se elaboró para la enseñanza de los métodos de cálculo mental.

2. El estudio experimental se enfocó hacia dos objetivos:

- La elaboración de una propuesta experimental de enseñanza de una selección relevante de los métodos de calculo mental como alternativa a la línea tradicional reflejada en los textos.

- Un análisis de los errores cometidos por los estudiantes de la muestra, antes y después de recibir enseñanza según la propuesta elaborada. El análisis se orientó hacia el estudio de su incidencia y tipologia, y hacia el establecimiento de una clasificación en torno a los mecanismos identificables de los procedimientos que los sustentan.

\section{EL PAPEL, DEL CÁLCULO MENTAL EN LOS CURRÍCULOS ACTUALES}

Usaremos la expresión cálculo mental para referirnos al cálculo de cabeza o de memoria (sin ayuda externa) con datos exactos. Algunos autores (Trafton, 1978) reservan 
esta denominación para los métodos alternativos, pero nosotros la tomaremos en el sentido más amplio, que incluye, por lo harto frecuente que es, la emulación y adaptación mental de los artificios estándar y los métodos de recuento.

El cálculo mental es un tema con interés educativo, ya que, aunque hay discrepancias en cuanto a la relativa importancia que debe concedérsele en el currículo, vuelve a ser incluido en la nueva propuesta oficial escolar española (DCB, 1989 y DBR, 1990). El enfoque actual no es el utilitario, rápido o agilizador đe la mente como antaño, sino otro basado en planteamientos que pretenden disminuir el énfasis tradicional sobre el cálculo escrito rígido para otorgárselo al calculo variado (mental, estimado, con calculadora o con algoritmos estándar según convenga). En este nuevo enfoque, se considera que el cálculo mental juega un papel en la adquisición de los conceptos relacionados con la operatoria, con la profundización de los conocimientos matemáticos intuitivos antes de su formalización y como medio para promover la reflexión de los estudiantes y la evaluación y reorientación del trabajo del profesor.

Las opiniones vertidas en la literatura puntualizan este nuevo papel del cálculo mental en el currículo con argumentos tales como los siguientes:

- Es un medio para incrementar la comprensión infantil de los números (Plunkett, 1979) individualizándolos y relacionándolos con diversas formas de escribirlos (Giménez y Girondo, 1990) y conociendo cómo está compuesto de sumandos y factores (Menchinskaya y Moro, 1975).

- Es de utilidad en la comprensión y desarrollo de los equivalentes escritos (Cockcroft, 1985), pudiendo llevar al descubrimiento de pautas, propiedades y estructuras de nuestro sistema numérico (Reys, 1985).

- Es un tipo de análisis de las situaciones numéricas que puede ser usado por los educadores para incrementar la flexibilidad en el cálculo (Henneessy et al., 1989) de los niños y su reflexión sobre el procedimiento mismo (French, 1977).

- Asimismo, se puede usar en el diagnóstico de la comprensión infantil del número y del valor de posición (Henneessy et al., 1989) y para comprobar las concepciones de los estudiantes ( $\mathrm{y}$ su disponibilidad) ligadas a la numeración decimal y a las propiedades de las operaciones (Butlen y Pezard, 1991).

- También es un dominio para introducir de modo informal ideas matemáticas que luego se desarrollarán más en profundidad (Cockcroft, 1985), para trabajar la transición del lenguaje vertical reglado o de columnas de la aritmética al horizontal simbólico del álgebra (Gómez, 1988) y, posiblemente, para desarrollar destrezas de resolución de problemas (Trafton, 1978; Polya, 1945)

Por todo esto, aunque las opiniones señaladas sugieren alternativas diferentes en cuanto a la finalidad de la enseñanza del cálculo mental, se puede decir, cuando menos, que el cálculo mental es valioso desde el punto de vista de la enseñanza-aprendizaje del pensamiento aritmético, y esto último sí que es algo en lo que hay acuerdo en cuanto a la posición relevante que debe ocupar en la educación obligatoria.

\section{LOS ERRORES Y SU IMPORTANCIA}

Tradicionalmente, los profesores han creído que los errores de cálculo que cometían los estudiantes eran debidos a una falta de dominio de los métodos o a un despiste a lo largo del proceso de cálculo. En esta postura, de corte conductista, se consideraba que los errores carecian de interés, eran algo que había que ignorar.

En la actualidad, algunos expertos en educación matemática (Menchinskaya y Moro, 1975; Radatz, 1979; 1980; Movshovitz-Hadar et al. 1987; Brousseau, 1983; Brousseau, Davis y Werner, 1986; Confrey, 1991; Borasi, 1987, 1994) tienen otra opinión en la que, en coherencia con el punto de vista constructivista del aprendizaje, se reconoce lo valioso que puede ser analizar los errores de los estudiantes. En este sentido, de acuerdo con Borasi (1994), el punto de vista dominante es el relacio. nado con el diagnóstico de las dificultades de aprendizaje y las sugerencias para remediarlas. Otras posturas (Confrey, 1991; Borasi, 1994) apuestan por enfoques en los que se busca explotar los errores para generar nuevas cuestiones y exploraciones o como trampolín para la indagación en matemáticas.

De acuerdo con Ia postura constructivista y en un enfoque de diagnóstico y remedio, Ios errores son una fuente de información para el profesor acerca de lo que han aprendido los estudiantes y cómo lo han aprendido (Borasi, 1994). Es más, son el síntoma indicativo de alguna patología subyacente, un método falso que el estudiante cree correcto (Brousseau, Davis y Werner, 1986), el efecto de un conocimiento anterior, que tenía su interés, su éxito, pero que ahora se revela falso o simplemente inadaptado. Los errores de este tipo no son errấticos e imprevisibles, se constituyen en obstáculos (Brousseau, 1983). Por tanto, un error no es sólo la ausencia de respuesta correcta, ni el resultado de un accidente; sino que es más bien un producto de la experiencia previa (Radatz, 1980), una parte del proceso de aprendizaje que se manifiesta de forma persistente y reproducible. También se considera (Menchinskaya y Moro, 1975) que los errores son el resultado de un proceso definido, cuya naturaleza puede ser desenmascarada.

Desde esta perspectiva, el análisis de los errores es muy interesante ya que puede revelar la existencia de obstáculos didácticos, modelos implícitos y dificultades individuales que están relacionadas con malentendidos instalados y consolidados, que la enseñanza práctica no siempre tiene en cuenta. 
«Los errores que no se deben a distracciones sino que se reproducen sistemáticamente en situaciones similares son muy interesantes porque nos revelan la existencia de modelos implícitos erróneos. Estos errores no aparecen aislados, sino que están relacionados con una cierta manera de conocer que permite detectar las resistencias a la evolución de un concepto, esto es, los obstáculos epistemológicos. Es de desear que los modelos implícitos erróneos se hagan explícitos produciendo errores que, en el decir de Anna Krygowska, podemos calificar de «errores benditos", porque nos ponen sobre la pista de malentendidos que se instalan y se consolidan si no se muestran explícitamente» (Centeno, 1988).

Cuando el análisis está hecho por el aprendiz, se vuelve una ayuda para su aprendizaje; y cuando está hecho por el profesor, es una ayuda para que éste diseñe una instrucción más eficaz.

En este sentido, para hacer emerger errores y abordar el análisis que permita desenmascarar su naturaleza y también para enfrentar a los estudiantes con su propio proceso de aprendizaje, el cálculo mental es un dominio aritmético privilegiado (Butlen y Pezard, 1991). Esto es así puesto que es constatable (Reys, Trafton, Reys, Zawojewski, 1984, cit. Reys, 1985; Gómez, 1994) que la falta de competencia de los estudiantes es general y sobretodo se constata cuando se pasa de calcular con números naturales a caicular con números decimales.

Antecedentes que sustentan el análisis de errores: categorías de las respuestas incorrectas en el cálculo.

En un estudio precedente (Menchinskaya y Moro, 1975) se señala que en el cálculo se pueden dividir las respuestas incorrectas en dos categorías básicas, según sea la fuente de las mismas: las que se derivan de las condiciones con que se llevan a cabo las operaciones y las que se derivan de la calidad del dominio de los conocimientos aritméticos.

Las primeras son mecánicas, surgen cuando bajo ciertas condiciones (cansancio, falta de interés, distracción, nerviosismo, etc.) el estudiante pierde el control consciente en la resolución del problema, por lo tanto no implican una falta de conocimiento y son esporádicas, ya que un ejemplo resuelto incorrectamente una vez puede ser resuelto correctamente una segunda vez, incluso sin que medie la corrección del mismo de una a la otra vez.

En esta categoría de fallos los autores incluyen entre otros el denominado perseverativo en el cual un número queda retenido fijamente en el pensamiento, como por ejemplo en $43+7=70$. También incluyen en esta categoría el que llaman inercia de la acción, en el cual se ejecuta una operación que no corresponde con el signo, tal vez condicionada por la influencia de una operación precedente, como, por ejemplo, cuando en una sucesión de sumas hay una resta intercalada que es resuelta incorrectamente como suma.

En la otra categoría de fallos, los autores distinguen dos subcategorías: una primera que agrupa los fallos basa- dos en una memorización pobremente establecida de determinados hechos numéricos, y una segunda que agrupa los fallos que se basan en la forma en que han sido aprendidas las reglas. En el primer caso, ponen como ejemplo las respuestas del tipo $7 \times 8=54$ o 58 y, en el segundo, las del tipo $1000: 200=50 \circ 500$, y $96: 16=10$

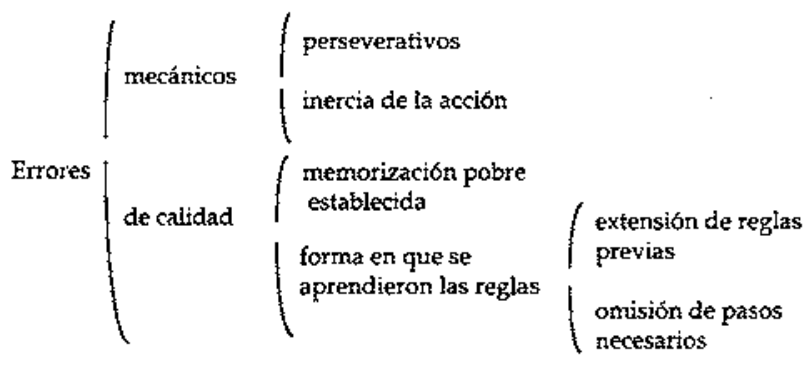

Este tlltimo tipo de respuestas incorrectas son las que interesan en este trabajo. En la medida en que son persistentes y reproducibles, las identificamos en este trabajo con los denominados errores sistemáticos de procedimiento.

No obstante la dificultad que tiene dar una definición de error sistemático de procedimiento, por cuanto ésta depende del enfoque en que va a ser utilizado y del contexto de la investigación, en este trabajo se la considera de un modo restringido, en el sentido de procedimiento inapropiado, persistente y reproducible que no se debe a distracción o inadvertencia, casualidad o fallo de memoria.

\section{MODELO TEÓRICO EXPLICATIVO DE LOS ERRORES}

Para explicar los errores debidos a la forma en que se aprendieron las reglas, los autores sugieren, a partir de sis observaciones en tests escritos con estudiantes de los grados $2^{\circ}$ y $3^{\circ}$ de una escuela de Moscú, que estos errores se basan en que hay reglas previamente aprendidas, cuyo campo de aplicación se extiende injustificadamente, o en las que se omiten determinados pasos necesarios, dando como resultado que la regla se lleve a casos donde no funciona.

Así, para ellos, el origen de la respuesta $1000: 200=50$ o 500 es la regla para sumar o restar números acabados en ceros, en donde se opera separando y bajando los ceros finales, que los estudiantes aplican de la misma manera al pasar a la división. La respuesta $96: 16=10$, surge análogamente, $90: 10=9 ; 6: 6=1 ; 9+1=10$. De nuevo lá regla aplicable para la suma y resta del cálculo escrito (en donde se operan por separado las cifras de las unidades y las de las decenas) se lleva a la división.

Esta explicación entronca con la que se ha dado para determinados errores en otras partes de la matemática, como la de Matz (1982) sobre los ertores sistemáticos en 
la resolución de problemas algebraicos. Para Matz, estos errores son el resultado de adaptaciones razonables, pero que no siempre funcionan, de los conocimientos previos adquiridos a nuevas situaciones, y algunos de estos errores del álgebra tienen su origen en una falta de dominio y comprensión de los procedimientos aritméti$\cos$.

Brousseau (cit. Palarea y Socas, 1994), señala que «los errores de este tipo se deben a que los alumnos usan inadecuadamente una fórmula o regla conocida, que han extraído de un prototipo o libro de texto, y la usan tal cual la conocen o la adaptan incorrectamente a una situación nueva. Tienden así un "puente" para cubrir el vacióo entre reglas conocidas y problemas no familiares".

Estas explicaciones están ligadas entre sí por un principio común. Este principio puede enunciarse, tomando las palabras de Brousseau (1983), cuando decimos que en los errores hay unna manera de conocer, una concepción característica, coherente ya que no correcta, un conocimiento antiguo y que ha tenido éxito en un dominio de acciones".

Por otro lado, Resnick (1992) plantea que los procesos de razonamiento por los que los niños inventan procedimientos incorrectos tienen su origen en la separación entre la representación cuantitativa de los símbolos (Ia semántica), que decae, y las reglas para la manipulación de los símbolos (la sintaxis), que predomina.

\section{PLANTEAMIENTO Y METODOLOGÍA DE LA PARTE EXPERIMENTAL}

\section{Objetivos}

Uno de los objetivos de este trabajo ha sido ver hasta qué punto este modelo explicativo es generalizable a los errores de procedimiento en cálculo mental y si además lo es con estudiantes de nivel superior, adultos, que han superado la edad escolar, e incluso que no sólo han demostrado su suficiencia en las nociones curriculares de aritmética y álgebra sino que se espera de ellos que en el futuro sean capaces de enseäarlas.

Para ello, inspirándose en las ideas anteriores, se ha tratado de hacer un análisis de los errores en cálculo mental con el fin de conocer su incidencia, su tipología y establecer una clasificación en torno a la identificación de mecanismos que puedan ser interpretados como los que se usan para establecer los puentes entre las reglas conocidas y las que las situaciones de cálculo mental planteadas demandan.

\section{Metodología}

El estudio se hizo con 144 estudiantes españoles de la Escuela de Magisterio de la Universitat de València a lo largo del curso académico 1992-93, en su ambiente universitario natural.
Los estudiantes resolvieron dos pruebas del tipo test con ejercicios de cálculo mental con números naturales y decimales. Entre el pretest y el postest se llevó a cabo una fase de enseñanza de acuerdo con la propuesta experimental elaborada (Gómez, 1994), que se comenta más adelante.

Los ejercicios de las pruebas fueron elegidos tras experiencias piloto atendiendo a consideraciones sobre su no excesiva dificultad, su vinculación a métodos alternativos, la posibilidad de hacer comparaciones entre los datos con números naturales o decimales, y que el número de ejercicios de cada prueba estuviera dentro de los límites razonables para que los estudiantes pudieran resolverlos en sesiones de una hora.

Se presentaron 14 ejercicios en la primera prueba y 20 en la segunda, la mitad de restar y la mitad de multiplicar. Los ejercicios se tenían que resolver mentalmente y, aunque no se limitaba el tiempo, sólo se disponía de una hora, lo que se reveló suficiente. Estos ejercicios se recogen en las tablas I y 11 .

Tabla I

Ejercicios del prestest.

\begin{tabular}{|c|c|c|c|c|c|c|}
\hline 547.189 & 243.75 & $1300-875$ & 461.166 & $265-199$ & $13 \cdot 8^{\prime} 75$ & $223-1 \cdot 58$ \\
\hline $3^{\prime} 1^{1}$ & $37 \times 025$ & $28 \times 35$ & $47 \times 99$ & $41 \times 42$ & $3^{\prime} 4 \times 0^{\prime} ! 5$ & $64 \times 25$ \\
\hline
\end{tabular}

Tabla II

Ejercicios del Postest.

\begin{tabular}{|c|c|c|c|c|c|c|c|c|c|}
\hline 737.289 & 634.75 & $148 x \cdot 675$ & 481.286 & 245.197 & 14.775 & 232.187 & $56^{\prime} 7+189$ & 243.75 & $46^{1} 1-166^{\prime}$ \\
\hline$A^{\prime} 1^{2}$ & $97 \times 4025$ & $26 \times 35$ & $37 \times 98$ & $31 \times 42$ & $24 \times 0^{\prime} 15$ & $66 \times 75$ & $28 \times 045$ & $49 x \leq 1$ & $19 x \times 18$ \\
\hline
\end{tabular}

El estilo fue escrito, es decir, que los ejercicios se presentaron en una hoja y se encareció a los estudiantes que escribieran exclusivamente el resultado, por lo que debian calcular mentalmente y no escribir nada hasta tener completado el resultado. Después había que explicar, en la misma hoja, cómo se había resuelto.

Esta información fue utilizada posterjormente para caracterizar tipos de error, mediante entrevistas individualizadas.

No se plantearon ejercicios de stumar y de dividir, porque se tenía el convencimiento, basado en experiencias piloto previas y en el análisis bibliográfico, de que la suma mental no fiene dificultades notables y la división apenas se hace mentalmente.

\section{La propuesta experimental de enseñanza}

La enseñanza consistió en la presentación a los estudiantes de una selección de los métodos de cálculo mental extraídos a partir del análisis de la literatura, durante un 
período de unas cuatro semanas, en el ambiente natural de clase de la asignatura de matemáticas, cuyo horario era de 3 horas por semana (En el anexo se describen estos métodos). En esta fase, de unas doce sesiones de 50 minutos aproximadamente durante el curso 1992-93, dedicada a la enseñanza del cálculo en general, los ejercicios fueron exclusivamente con números naturales.

La enseñanza se configuró atendiendo a una propuesta experimental que pretendió ofrecer una alternativa al modelo dominante en los manuales escolares en vigor, que es de carácter reglada, de adiestramiento repetitivo y rígido: «para este ejemplo, este método».

La propuesta se sustentó en una breve presentación con ejemplos numéricos de acuerdo con los principios siguientes:

- Una secuencia de presentación de los métodos en tres etapas. A saber: métodos para números de una cifra, métodos para números de dos cifras y viejas reglas que historicamente han coexistido con los algoritmos estándar.

- La formalización de los métodos en el lenguaje horizontal simbólico del álgebra, algunos incluso mediante representaciones literales: $(10 a+b) \times(10 c+d)=\ldots$, etc.

- El «diálogo de resoluciones», esto es, la verbalización de los distintos métodos usados por los estudiantes sobre un mismo ejercicio puesto en la pizarra para ser resuelto libremente, y discusión de su campo de validez, ventajas e inconvenientes, de lo que tienen en común y diferente, de las leyes que los rigen y de los principios directores o estrategias que los sustentan.

- Una organización de los métodos en forma de esquema estructurado que los sintetiza y compendia, a partir de sus características unificadoras y globalizadoras, para evitar una presentación exhaustiva, que no estaría justificada por el papel preponderante que le daría al cálculo mental ni, por otra parte, sería posible debido al escaso tiempo escolar disponible.

\section{RESULTADOS DEL ESTUDIO EXPERI- MENTAL}

1. Como consecuencia de la enseñanza, se pasó de una situación en la que la mayoría de los estudiantes usaron exclusivamente métodos de columnas, a una situación en la que la mayoría usaron preferentemente métodos alternativos. La tabla III muestra esta variación en términos de porcentajes de estudiantes.

Esto trajo consigo la aparición de una amplia tipología de errores, debida a la multitud de procedimientos inapropiados empleados por los estudiantes.

Aunque la mayor o menor frecuencia de los tipos de errores observados guardó relación con las características de los ejercicios (p. e., fue mayor con los decimales que con los enteros) y de los métodos empleados (p. e., redondeo o descomposición) se evidenciaron ciertas tendencias, ya que hubo algunos errores que fueron especialmente frecuentes.

En particular:

- Restar, en vez de sumar, el parcial que compensa el efecto del redondeo del sustraendo ( 24 de los 48 errores de procedimiento en la resta fueron de este tipo).

Ejemplo: $245-197=48 . \ll 245-200=45-3=42 . »$

- Omitir los productos parciales en cruz en la doble descomposición (39 de los 188 errores de procedimiento en la multiplicación fueron de este tipo).

Ejemplo: $28 \times 35=640$. «He multiplicado $20 \times 30=600$ y después $8 \times 5=40$ y he sumado $600+40=640$."

- Multiplicar los datos que resultan al añadir a uno de los dados lo que se le quita al otro (26 de los 188 ).

Ejemplo: $64 \times 75=4830$. «Al 75 le quito $5, y$ al 64 le pongo 5 , de manera que queda $69 \times 70 . »$

- Desplazar la coma decimal hasta su eliminación en un dito (20 de 188).

Ejemplo: $3{ }^{\prime} 4 \mathrm{x}^{\prime} 15=10^{\prime} 5$. «He multiplicado 34 por $1^{\prime 5} \mathrm{y}$ wie da $6^{\prime} 0+4^{\prime} 5=10^{\prime} 5$.»

- Recuperar inadecuadamente la coma decimal (19 de 188).

Fjemplo: $37 \times 0^{\prime} 25=0^{\prime} 925$. «Multiplico $5 \times 37=185$ y $2 \times 37=74$. Sumo $185+740=925$, luego añado el cero a la izquierda 0'925."

La tabla IV muestra la elevada incidencia relativa de los errores sistemáticos de procedimiento. Obsérvese que

Tabla IIt

\begin{tabular}{|l|c|c|c|c|c|}
\hline $\begin{array}{l}\text { Sólo usaron métodios } \\
\text { de columnas }\end{array}$ & Resta & Multiplicación & $\begin{array}{l}\text { Usaron otros métodos más } \\
\text { de la mitad de veces }\end{array}$ & Resta & MuItiplicación \\
\hline Pretest & 62,5 & $63 \%$ & pretest & $16 \%$ & $21 \%$ \\
\hline Postest & $4,5 \%$ & $6 \%$ & Postest & $80 \%$ & $80 \%$ \\
\hline
\end{tabular}


en la multiplicación se contabilizaron 188 errores de procedimiento sobre 103 errores mecánicos y 66 respuestas en blanco, y que en la sustracción fueron 48 errores de procedimiento sobre 53 mecánicos.

Tabla IV

Frecuencia de errores.

\begin{tabular}{|lccc|ccc|}
\hline \multicolumn{3}{|c|}{ En la resta } & & \multicolumn{3}{c|}{ En la multiplicación } \\
\hline Errores & Naturales & Decimaltes & Total & Naturales & Decimales & To19l \\
\hline Mecánicos & 22 & 31 & 53 & 45 & 58 & 103 \\
\hline De procectimiento & 24 & 24 & 48 & 63 & 125 & 188 \\
\hline Resp. en blanco & 0 & 3 & 3 & 14 & 52 & 66 \\
\hline No descifrados & 3 & 4 & 7 & 6 & 6 & 12 \\
\hline Totales & 49 & 62 & 111 & 128 & 241 & 369 \\
\hline
\end{tabular}

De la misma manera que el análisis bibliográfico evidenció que los métodos y estrategias para el cálculo mental con números decimales eran los mismos que para el cálcuio mental con números naturales, también lo evidenció el desempeño de los estudiantes, los cuales aplicaron en los ejercicios con números naturales los mismos métodos que también aplicaron en los ejercicios con números decimales, y viceversa.

2. Se observaron dos tendencias diferenciadas en cuanto a la forma de operar los ejercicios con números decimales en la resta y en la multiplicación, que están de acuerdo con los hábitos del cálculo escrito.

En la resta con números decimales, la tendencia fue aplicar los métodos alternativos sin quitar la coma decimal. Mientras que en la multiplicación ocurrió al revés, la tendencia fue prescindir de la coma decimal, bien reduciendo los datos decimales a números naturales mediante alguna expresión equivalente (p. e., $0^{\prime} 25=1 / 4$ ), o bien usando la regla del algoritmo de columnas que consiste en colocarla según indique la suma del número de cifras decimales de cada uno de los datos.

En la multiplicación, algunos estudiantes evidenciaron dificultades para transferir los métodos aprendidos con los números naturales a los ejercicios con números decimales. Éstos, del orden de un $12 \%$, usaron métodos alternativos en los ejercicios con números naturales, pero no en los ejercicios con los decimales. Preguntados en las entrevistas manifestaron que creían que al prescindir de la coma decimal los datos que se obtendrían serían otros diferentes a los dados y, por lo tanto, tendrían un producto diferente, con cifras diferentes. No obstante, estos mismos estudiantes sospechaban que tenían que quitar la coma, pero no lo hacían porque no sabían cómo recuperarla después, ya que la regla aprendida que se basa en contar el número de cifras decimales de los datos la vinculaban rígidamente al algoritmo estándar de columnas.

3. Los estudiantes evidenciaron una falta de percepción de la sintaxis de los procedimientos en el lenguaje horizontal de paréntesis e igualdades del álgebra. Esto probablemente les hubiera evitado errores al enfrentar- les con aquéllos de sus procedimientos que no eran consistentes.

En efecto, el estudiante que resolvió diciendo $31 \times 42=$ "Multiplico $30 \times 42$ y Ie sumo 31 » conocía la sintaxis de la propiedad distributiva y era capaz de reconocerla en su forma usual en los textos, $3 \times(4+5)=3 \times 4+3 \times 5$, pero no fue capaz de representarse la situación de esta manera: $31 \times 42=(30+1) \times 42$.

4. Del análisis de las respuestas de los estudiantes en los tests y con ayuda de entrevistas individuales se identificaron tres mecanismos que sustentan los procedimientos incorrectos de los estudiantes. Éstos son:

a) Extrapolaciones. Inserciones improcedentes de alguna parte o de algunos de los pasos, pero no de todos, de las reglas aprendidas, que los estudiantes extraen y llevan a otra regla o método que están aplicando en una situación nueva en la que no funcionan.

b) Generalizaciones. Extensiones de métodos completos o reglas que los alumnos aplican, tal cual las conocen o han aprendido, a situaciones nuevas en las que no funcionan.

c) Centramientos. Son métodos que sufren una interferencia por algún hecho que centra la atención del resolutor provocando que algún paso o resultado intermedio se desvíe de su aplicación correcta.

A continuación se ilustran estos mecanismos con un ejemplo escogido de cada uno de ellos. Estos ejemplos se describen transcribiendo literalmente en entrecomillado la descripción que los mismos estudiantes anotaron en su hojas del test, como explicación de lo que habían hecho mentalmente. Después se presenta un extracto de la entrevista que se le hizo al estudiante, insertándose entre paréntesis en el texto de la entrevista notas aclaratorias de la conversación.

\section{Extrapolaciones}

César cometió el siguiente error en un ejercicio de multiplicar:

$$
\begin{aligned}
& 2^{\prime} 4 \times 0^{\prime} 15=36 . \ll 2^{\prime} 4 \times 0^{\prime} 15=240 \times 15=240 \times 10=2400,2400+ \\
& \frac{1}{2} 2400=2400+1200=3600 \rightarrow 36^{\prime} 00 » .
\end{aligned}
$$

La entrevista reveló que César se fija en el dato que más cifras decimales tiene, en este caso 0'15. Como éste tiene dos cifras decimales, para eliminar la coma decimal multiplica por 100 tanto 0 ' 15 como 2'4, obteniendo $240 \times 15$.

Esta manera de suprimir la coma decimal es válida en la división cuando el divisor es decimal pero aquí no funciona. César parece haber extrapolado este paso de la división al caso de la multiplicación.

Profesor.- ¿Quieres volver a hacer este ejercicio? ¿Qué has hecho? 
César.- Multiplico por 100 las dos partes.

P. ¿ ¿Para qué?

C.-Para quitar, de esta forma, aquí el decimal (señala el 0'15).

P. - ¿A los dos?

C.- Me interesa más el de la derecha.

P.- ¿Y luego qué pasa?

[...]

P.- ¿Por qué multiplicas por cien los dos (datos)?

C.- ¿Por qué multiplico por 100 ? Para obtener aquí también... quitar.

P.m (Intertumpiendo) ¿Para quitar la coma?

C.- Para quitar la coma.

P.- ¿En los dos factores?

C. - Me interesa más el de la derecha.

P. ¿ ¿Por qué te interesa más el de la derecha?

C.- Pues para tener unidades y quitarle después la coma.

P.- ¿Al de la derecha?

C.-Sí.

P. - ¿Al de la izquierda no?

C. - Al de la izquierda ya no se lo quito, porque, si quito dos a la derecha (se refiere a las dos cifras decimales de 0 '15), tengo uno a la izquierda (se refiere a la cifra decimal de $2^{\prime} 4$ ), que ya es seguro que lo voy a quitar.

P.-En realidad, $i$ el que miras es el que tiene más cifras?

C.- Por supuesto.

P.- ¿No porque sea el de la derecha?

C.- No, no, no; si hubiera sido el de la izquierda el que tiene más, siempre miro el que más decimales tiene.

[ ...]

P.-Vamos a ver. ¿Cuántos decimales hay (en 2'4 $\mathrm{x}^{\prime}$ '15)?

C. -3 . Tiene que haber 3 !

P.- Y al final, en el resultado ¿cuántos crees que tiene que haber?

C. $-; 2$ !
[...]

P. - $i Y$ ahora qué estas pensando?

P.- Pues nada, que no había que multiplicar por 100 , sino simplemente bacer la operación, luego poner las comas.

P.-- Es verdad. ¿Y por qué multiplicabas por 100 ?

C.-- Pues no sé.

P.- ¿Está mal?, ¿tú crees que multiplicar por 100 está mal?

C.-- Yo creo que no. Es correcto si luego quitas dos (dos ceros del resultado final). Sí.

P.- ¿ Por qué crees que es correcto?

C.- Porque tú cuando multiplicas los dos factores de una multiplicación, el resultado, luego, es el mismo, no es proporcional. [ ... ]

P. ¿ ¿Por qué pensabas que tenías que multiplicar por 100 ?

C.- ¿Por qué veía esto? Veía dos cifras decimales.

[...]

P. - ¿Para qué has querido multiplicar por 100 ?

C. - Es que... ¿No estaré yo confundido con la división?

P.- Algo de la división hay ahí, ¿verdad?

C.-Claro. Por ejemplo. No sé... 160 entre 30 quito esto, un cero de aquí y otro de aquí.

P.- - X si fuera con decimales? Pon la división 2'4:0'15.

C. - Pues, ¿qué haría?, quito una de aquí y corro ésta un lugar (le da 24:1,5).

P.- Pero no has terminado. Aún no puedes dividir.

C.- Claro. Entonces aqui quedaría uno (una cifra decimal) y pondría un cero (le da $240: 15$ ).

P. ¿ ¿ eso? ¿Qué operación has hecho en realidad?

C.- Multiplicar.

P. ¿Multiplicar por cuánto?

C.- Por 100.

P. - ¿Dónde?

C. - A los dos lados.

P.-Entonces, ¿crees que es eso lo que estabas pensando? 
C.- Inconscientemente lo estaba pensando, lo que pasa es que no te lo quería decir porque no sabía si...

P.- Ah! iYa lo pensabas. Lo has estado pensando todo el rato!

C. - No, todo el rato, no. O sea, lo tenía en cuenta, sabía que había algo ahí por el cual yo estaba haciendo eso y me estaba dando cuenta.

\section{Generalizaciones}

Beatriz cometió el siguiente error:

$245-197=245-200=45-3=42$.

La entrevista reveló que Beatriz extiende el método de compensación, que es válido en la suma, por el cual, si se aumenta un sumando, después hay que restar la misma cantidad al resultado. Parece que generaliza al caso de la resta porque no entiende que esto pueda ser diferente: "lo que pasa es que no sé por qué en la suma sí y en la resta no».

P.- ¿Quieres explicar to que has hecho?

B.- He sumado (3 a 197) para que me sea más fácil restar. ¿Entonces qué hago? Le he quitado a 245 las 200 y da 45 . Hasta ahí está bien.

P.- Sí.

B.- Pero si he sumado... ¡Claro! Ahora tengo que restar. Si le sumo, resto.

P.- ¿Para qué?

B. Para quitar la (interrumpe). Bueno, yo he sumado para igualarlo. Ahora le tengo que restar la misma cantidad que he sumado para que quede bien el resultado.

\section{Centramientos}

Amparo cometió el siguiente error: $19 \times 18=341$. «20x18 $=360 ; 19 \times 1=19 ; 360-19 \approx 341$."

La entrevista reveló que Amparo fija su atención en la necesidad de compensar la alteración efectuada al redondear el 19 a 20 , pero viola el significado de la multiplicación $19 \times 18=(20-1) \times 18$, ya que se centra en que es con el número alterado, el 19, con el que debe disminuirse el producto.

Amparo.- Y luego, como me había pasado... (se refiere a que al multiplicar $20 \times 18$ se ha pasado en el resultado). O sea, como de 19 a 20 va uno solo, pues he multiplicado 19 , que es el número que a mí me dan, por 1 , porque me faltaba 1. Entonces me da 19 y, como en la primera multiplicación me pasaba del resultado que me tenía que dar, porque 20 era un número mayor que 19 , pues entonces lo he restado para saber lo que me daba.
(Tras otros comentarios)

P. $\rightarrow$ ¿Restaste el 19 ?

A.- Porque como lo había redondeado al 20.

P.- Te creías que hay que quitar el mismo que has redondeado.

A.-Sí. Porque es que el 20 es con el que... O sea, a causa del 20 yo me paso en la multiplicación, y no pasa con el 18. El 18 yo no lo muevo, lo dejo como está. Entonces, para saber de cuántos veintes me he pasado...

P. - (Interrumpiendo) $¿$ De qué te has pasado, de veintes o de dieciochos?

A.- De veintes.

\section{CONCLUSIONES}

1. El análisis de los errores ha permitido hacer explícitos algunos malentendidos acerca de los procedimientos de cálculo, que de otra manera, cuando sólo se aplicaban los métodos estándar, como en el pretest, no pudieron emerger. De esta forma, se evidencia que los métodos históricos de cálculo mental son un dominio privilegiado para hacer emerger una problemática ligada al aprendizaje de la aritmética que, de otra manera, mientras sólo se calcula con los métodos de columnas, permanece oculta.

Además, se han podido contrastar las concepciones de los estudiantes, lo cual ha permitido conducirles hacia la reconceptualización de sus nociones aritméticas. Esto se ha hecho confrontando a los estudiantes con sus formas inapropiadas de resolución, por medio de entrevistas individualizadas, en las que se desencadenaron procesos cognitivos sobre la base de una mejor comprensión del significado y de las propiedades de las operaciones, del uso de las nociones del sistema de numeración, de las expresiones numéricas equivalentes, de la representación simbólica del lenguaje horizontal de ecuaciones y paréntesis, y del efecto invariante en el resultado de ciertas alteraciones de los datos.

Los resultados obtenidos concuerdan con los establecidos con alumnos más jóvenes por otros autores ya señalados, como esperábamos, en cuanto a que determinados errores de procedimiento son adaptaciones a nuevas situaciones de los conocimientos previos adquiridos.

La problemática identificada parece que concuerda tambiên con la hipótesis que plantea que los procesos de razonamiento por los que los niños inventan procedimientos incorrectos tienen relación con la separación entre la sintaxis y la semántica subyacente.

2. La investigación permite concluir que el modelo teórico explicativo de los errores descrito anteriormente es lo bastante general para explicar to que ocurre en el 
dominio del cálculo mental y con estudiantes de nivel educativo superior.

Del análisis efectuado se sigue que los estudiantes utilizan procedimientos inapropiados en los cuales los mecanismos que se han identificado son: generalizaciones, extrapolaciones y centramientos, los cuales estarían relacionados con la influencia de los conocimientos previos sobre los sobrevenidos.

Unas veces, estos mecanismos parecen debidos a la sobregeneralización de propiedades no explicitadas de determinadas situaciones numéricas, que ellos perciben como verdaderas porque les han funcionado en un determinado campo de validez, pero que no es cierto que siempre funcionen. Otras veces, parece que son debidos a rigideces motivadas por un predominio de lo sintáctico sobre lo semántico. En otras palabras, predomina la atención en lo reglado sobre el significado de las reglas y en lo alterado sobre el efecto de las alteración en los resultados.

3. En general, a lo largo de las pruebas los estudiantes no reconocieron, ni aprovecharon las ventajas de expresarse en el lenguaje simbólico de ecuaciones y paréntesis del álgebra y prefírieron usar el lenguaje retórico y reglado de la aritmética. Probablemente muchos de sus errores se hubieran evitado si así lo hubieran hecho.

4. La enseñanza de los métodos histórico-intrínsecos de cálculo mental para los números naturales produjo su uso no sólo con estos números sino también con los decimales, salvo en aquellos estudiantes, los menos, que vincularon las reglas para operar la coma decimal exclusivamente a los algoritmos estándar del formato vertical de columnas en vez de al cálculo con decimales en general.

5. También se ha observado que los estudiantes no hicieron valoraciones sobre la razonabilidad o no de sus resultados incorrectos.

En consecuencia, cabe pensar que estamos ante un producto de una enseñanza excesivamente orientada al automatismo en el manejo de la notación simbólica, en detrimento de la reflexión sobre las cantidades en juego y las relaciones que esa notación expresa, así como sobre los principios en los que se basan los procedimientos y el efecto que las alteraciones en los datos produce en los resultados.

\section{Implicaciones educativas}

Se ha presentado, de manera resumida, una investigación en relación con el cálculo mental que pone de manifiesto problemas en la enseñanza que permanecen ocultos cuando sólo se trabaja con los algoritmos de lápiz y papel.

Esta problemática evidencia en muchos estudiantes una comprensión pobre del efecto que las alteraciones en los datos produce en los resultados, un débil reconocimiento de los conceptos, leyes y principios que rigen la operatoria, y una ausencia de la necesaria comprobación o estimación de la razonabilidad del resultado que, en caso de discrepancia con el resultado primeramente obtenido, podría haberles hecho recapacitar sobre su forma de proceder.

Los métodos de cálculo mental tal y como se han utilizado en este trabajo, se han revelado especialmente valiosos para:

- El desarrollo del pensamiento aritmético, en particular lo relacionado con el análisis de las situaciones numéricas y el manejo de los hechos de la numeración para la expresión flexible, significativa y no automática de las acciones sobre los números.

- El diagnóstico, tanto para que el profesor conozca las concepciones que sobre los procedimientos de cálculo tienen los estudiantes como para que ellos se vean obligados a enfrentarse con ellas, sentando así las bases para su posible reconceptualizacion.

En este aspecto, destaca la importancia de hacer emerger los errores de los estudiantes como ayuda para conocer sus creencias, la forma en que están aprendiendo o han aprendido, las difícultades que enfrentan. Este conocimiento es provechoso para los profesores, para que puedan desarrollar una instrucción más efectiva, insistiendo en aquellos aspectos en los que los estudiantes pueden cometer errores, anticipando las respuestas de los alumnos y diseáando estrategias para la corrección de las mismas cuando se requiera.

Conviene tener esto presente, ya que, como se ha señalado (Palarea y Socas, 1994), la superación de los errores por parte de los alumnos constituye un tema básico en el aprendizaje que genera grandes dificultades. Las investigaciones actuales señalan que los errores están profundamente interiorizados por los alumnos y que no son de fácil eliminación. Incluso, en muchos casos, parece que los estudiantes han superado un error $y$ luego lo vemos, con desilusión, resurgir al poco tiempo. Para ello, plantear a los estudiantes que su comprensión conceptual es incorrecta y darles entonces una explicación es, a menudo, insuficiente para eliminar el error.

Mi experiencia, como resultado de esta investigación, me dice que el camino que debemos emprender pasa necesariamente por enfrentar a los alumnos con sus propios errores, provocar el conflicto en su mente haciéndoles ver la inconsistencia de sus respuestas al pedirles comprobaciones y pruebas, hacerles ver las ventajas del lenguaje simbólico sobre el retórico para descubrir y reconocer las leyes y principios que está aplicando, y reflexionar sobre el efecto de las alteraciones de los datos sobre los resultados.

En este sentido es en el que deberían utilizarse los métodos de cálculo mental que recogen las nuevas orientaciones curriculares oficiales, de tal modo que al poner el énfasis en las propiedades, el lenguaje y las relaciones numéricas que sustentan los métodos se evite caer en los 
viejos hábitos, que ya hicieron que una vez el cálculo mental cayera en desuso.

En consecuencia, en la medida en que la problemática identificada en este trabajo indica la existencia de algunos efectos no deseados de una enseñanza escolar excesivamente volcada al automatismo en las operaciones y prácticamente centrada en los algoritmos de columnas,

\section{REFERENCIAS BIBLIOGRÁFICAS}

BORASI, R. (1987). Exploring mathematics through the analysis of etrors. For the Learning of Mathematics, 7, 3, pp. 2-8

BORASI, R. (1994). Capitalizing on errors as «springboards for inquiry" a teaching experiment. Journal for Research in Mathematics Education, 25(2), pp. 167-202.

BROUSSEAU, G. (1983). Les obstacles épistémologique et les problèmes en Mathématiques. Recherches en didactique des Mathématiques, 4(2), pp. 167-198.

BROUSSEAU, G., DAVIS, R.B. y WERNER, T. (1986). Observing Students at Work, en Christiansen, B., Howson, A.G. y Otte, M. (eds).Perspectives on Mathematics Education. Dordrecht: D. Reidel Publishing Co.

BUTLEN, D. y PEZARD M. (1991). Calcul mental, calcul rapide. Grand N., 47, pp. 35-59.

CENTENO, J. (1988). Numeros decimales ¿Por qué? ¿Para $q u e ́$ ? Madrid: Síntesis.

COKCKOFT, W.H.(1985). Lasmatemáticas sícuentan. Madrid. MEC. (Edición original: Mathematics Counts. Londres. HMSO, 1982).

CONFREY,J.(1991). Leaming to listen: A student'sunderstanding of powers of ten, en von Glasersfeld, E. (ed.), Radical constructivism in mathematics education, pp. 111-138. Dordrecht: Kluwer Academic Publishet.

D. B. R. (1990), Documento Base para la reforma de la EGB. Valencia: Conselleria de CuItura. E. y C. de la C. Valenciana.

D. C. B. (1989). Documento Diseño curricular base. Mađrid: MEC.

FRENCH, D. (1977). Mental methods in mathematics. Mathematics in School, 16(2), pp. 39-41.

GIMÉNEZ, J. y GIRONDO, L. (1990). Càlcul a l'escola. Barcelona: Graó.

GÓMEZ, B. (1988). Numeración y Calculo. Madrid: Síntesis.

GÓMEZ, B. (1994). El cálculo mental en el contexto educativo: un análisis en la formación de profesores. Memoria de Tesis doctoral. Universitat de València-España.

HENNESSY, S. et al. (1989). An Intelligent Tutoring System Approach To Teaching Primary Mathematics. Educational Studies in Mathematics, 20(3), pp. 273-292.

MATZ, M. (1982). Towards a process model for high school algebra errors, ent Sleeman, D. y Brown, J.S. (eds.), Intelligent Tutoring Sistems, pp. 25-49. Londres: Academic Press. cabe postular la conveniencia de un cambio en la enseñanza que aproveche los métodos de cálculo mental, en la forma como se ha descrito aqui, para hacer emerger errores, sentar las bases para hacerlos desaparecer y conseguir una mejora de las concepciones que los estudiantes tiene sobre los procedimientos aritméticos como medio de expresión significativa y no automática de sus acciones sobre los números.

MENCHINSKAYA, N.A. y MORO, M.J. (1975). Instruction in mental and written calculation, en Kilpatrick, J., Wirszlup, J., Begle, E. y Wilson, J. (eds.), Soviet studies in the psychology of learning and teaching mathematics, 14 , pp.73-88. Standford, California: School Mathematics Study Group.

MOVSHOVITZ-HADAR, N., ZASLAVSKY, O. y INBAR, S. (1987). An empirical classification model for errors in high school mathematics. Journal for Research in Mathematics Education, 18(1), pp. 3-14.

PALAREA, M. y SOCAS, M. (1994). AJgunos obstáculos cognitivos en el aprendizaje del lenguaje algebraico. Suma, 16 , pp. $91-98$

PLUNKETT, S. (1979). Decomposition and All That Rot. Mathematics in School, 8(3), pp. 2-5.

POLYA, G. (1945). How to solve it. Princeton U. P. (Trad. cast., 1951, Cómoresolver y plantear problemas. México: Trillas).

RADATZ, H., (1979). Error analysis in mathematics education. Journal for Research in Mathematics Education, 10(3), pp. 163-172.

RADATZ, H. (1980). Students errors in the mathematical learning process. For the Learning of Mathematics, 1(1), pp. 16-20.

RESNICK, L. B. (1992). From Protoquantities to operators: Building mathematical competence on a foundation of everyday knowledge, en Leinhartdt, G., Putham, R. y Hattrup, R. (eds.), Analisis of Arithmetic for Mathematics Teaching, pp. 373-429. Hilisdale, New Jersey: LEA.

REYS, B.B.(1985). Identification and characterization of mental computation algorithms used by seventh and eighth grade students on visually and orally presented mental computation exercises. (Doctorald., U.of Missouri-Columbia).Dissertation Abstracts Int., $46,3279 \mathrm{~A}$.

REYS, B.J.,TRAFTON, P.R., REYS, R.E. y ZAWOJESKI, J. (1984). Developing computational estimation materials for the middle grades-final report, $N^{2}$ NSF8113601, National Institute of Education: Washington D. C. National Science Foundations (cit. Reys, 1985).

TRAFTON, P.R. (1978). Estimation and mental Arithmetic: Important Components of Computation, en Suydam, M,N. y Reys, R.E. (eds.), Developing Computational Skills: 1978 Yearbook, pp. 196-213. Reston, VA. NCTM. 


\section{ANEXO}

\section{Los métodos enseñados}

\section{ARTIFICIOS}

\section{Reglas}

Multiplicación reglada de números formados sólo por unos.

Multiplicación por 11.

$57 \times 11=$ «Dejo el 7 , sumo $5+7,12$, dejo el 2 y llevo 1 al 5 , que son 6 . Total 627 .

Multiplicación de 101 por un número de 2 cifras, y 1.001 por uno de tres.

$58 \times 101=\ll$ A 58 le añado 58 . Total 5858».

Multiplicación reglada de números formados sólo por nueves.

$47 \times 99=\ldots \ll 47-1,46.100-47,53$. Total 4653».

Multiplicación de números de dos cifras o tabla mayor. Son las reglas que resultan de agrupar factores comunes al aplicar la doble distribución a toda la castrística de los números comprendidos entre 10 y 100.

a. Los números son iguales en decenas.

$(10 a+b)(10 a+c)=10 a(10 a+b+c)+b c: 25 \times 27=(25+7) \times 2 \times 10+7 \times 5=675$.

b. Los números son iguales en decenas y sus unidades suman diez.

$(10 a+b)(10 a+c)=100 a(a+1)+b c: 47 \times 43=40 \times 50+3 \times 7=2021 ; 35 \times 35=\ll 12$ y añado $25 »=1225$.

c. Los números son iguaies en unidades y sus decenas suman diez.

$\left.(10 a+b)(10 c+b)=100(a c+b)+b^{2}\right): 34 \times 74=(3 \times 7+4) 100+4 \times 4=2516$.

Multiplicación por complementos. Consiste en apoyarse en el producto de los complementos aritméticos cuando éste es más fácil que el de los dados.

a. Multiplicación por la regla de «los perezosos»: $a b=(10-a)(10-b)+10[a-(10-b)])$.

$8 \times 7=\ll 10-8=2 ; 10-7=3 ; 8-3=5$, son las decenas, y $2 \times 3=6$ son las unidades. Total $56 »$.

b. Multiplicación por la regla de «San Andrés»; ab=(100-a) $(100-\mathrm{b})+100[\mathrm{a}-(100-\mathrm{b})]$.

$89 \times 98=\ll 100-98=2,100-89=11,98-11=87$ son las centenas, $2 \times 11=22$, son las unidades $»=8722$.

Multiplicación cruzada o cruceta. Consiste en sumar los parciales del mismo orden al tiempo que se obtienen, para ahorrarse filas, de las que salen en el algoritmo escrito.

$17 \times 16=\langle 6 \times 7,42,2$ y me lievo 4.6 y $7,13.13$ y 4 que me llevo 17,7 y me llevo 1 . Siempre 1 y I que me llevo son 2.

Total 272».

\section{Formulas}

Cuadráticas

a. Cuadrado de un número por la fórmula del binomio:

$31^{2}=(30+1)^{2}=30^{2}+2 \times 1 \times 30+1=961$.

b. Multiplicación de dos número en condiciones de cero central, por la fórmula de la diferencia de cuadrados: $a x b=([(a+b) /$ $2]^{2}-[(a-b) / 2]^{2}: 19 \times 21=20^{2}-1=399$. 


\section{Numéricas}

$1+2+3+4+5+6+7+8+9+10+\ldots+40=40 \times 41: 2=820 ; 61+63+65+67+69=5 \times 65=325$.

2. DESCOMPOSICIONES. Uso de cantidades más pequeñas que las cadas.

Disociaciones. Son las descomposiciones de uno o ambos datos en sumandos.

Disociaciones por descabezamiento. Son las disociaciones que resultan al completar las cifras con sus ceros correspondientes o, lo que es Io mismo, al descomponer en órdenes de unidad.

\section{De un dato}

a. Agregar: Suma sucesiva de los diversos ódenes de unidad de un sumando, empezando por el orden superior: $63+45=63 \div 40+5=108$.

b. Segregar: Resta sucesiva de los diversos órdenes de unidad deI sustraendo, empezando por el orden superion: $894-632=894-600-30-2=262$.

c. Distribuir: Multiplicación sucesiva de los diversos órdenes de unidad de un factor: $46 \times 32=(40+6) \times 32=40 \times 32+6 \times 32$.

De los dos datos

a. Resta descabezando los dos datos y recuperando: $725-443=((700-400)+25)-43$.

b. Suma descabezando los dos datos y reagrupando. $154+26=(150+20)+(4+6)$, o $15+2,17,170,4+6,10$. Total 180 .

Disociaciones subsidiarias. Son las disociaciones de un dato en función del otro.

a. Resta haciendo la misma terminación: $461-166=461-161-5=295$.

b. Resta prestando o pidiendo refuerzos del minuendo a una resta parcial. $13-8,25=(12-8)+(1-0,25)$.

c. Patrones-Hecho conocido: dobles, complementos, cuadrados $25+28=25+25+3=53 ; 54+48=54+46+2=102 ; 25 \times 26=25 \times(25+1)=650$.

Factorizaciones. Son las descomposiciones de uno o ambos datos en factores.

Multiplicación por $12,22,33,44 \ldots: 37 \times 12=37 \times 3 \times 4=111 \times 4=444 ; 26 \times 33=(26 \times 11) \times 3$.

3. COMPENSACIONES. Uso de cantidades mayores y menores que Ias dadas.

Compensación intermedia. Es servirse del incremento de uno o Ios dos datos compensando el resultado durante el proceso infermedio, antes de operar los parciales.

Añađir y quitar. Añadir a un dato las unidades que se le quitan al otro.

a. Suma completando decenas: $81+59=80+60$.

b. Suma doblando el número central, conocido (Green, 1985) como procedimiento del «número misterioso»: $34+36=35+35=70$.

Promediar. La media de otros dos productos formados de modo que uno de sus datos es el mismo que uno de los dados y los otros dos son números equidistantes del otro dado.

$60 \times 25=\ldots 60 \times 20=1200,60 \times 30=1800$. Luego $60 \times 25=(1200+1800) / 2=1500$. 
Doble y mitad. Consiste en doblar un dato y dimidiar el otro.

a. Multiplicación por $15,35,45,55,65,75,85,95$, por un número par: $28 \times 35=14 \times 70=980$.

b. Multiplicación por un número que es potencia de 2 : $16 \times 36=8 \times 72=4 \times 144=2 \times 288=576$.

Conservar. Sustraer sumando o restando el mismo número al minuendo y al sustraendo para hacer decenas, centenas... completas. Resta sumando a los dos datos el complemento del sustraendo: $46-18=48-20=28$.

Alicuotar. Aplicar una relación alícuota, esto es, una relación de «ser divisor de». En particular, de la unidad seguida de ceros o de múltiplo de la misma.

Multiplicación por $5,25,125,75: 420 \times 5=420 \times 10 / 2 ; 64 \times 25=64 \times 100 / 4=1600 ; 36 \times 75=(36: 4) \times 3 \times 100=2700 ; 72 \times 125=72 \times 1000 /$ $8=9000$.

Compensaciones finales. Es servirse del incremento de tno o los dos datos, compensando el resultado al acabar las operaciones parciales.

Redondeo. Completar la decena, centena... inmediata superior de alguno de los datos.

a. Suma añadiendo unidades a cualquiera de los sumandos para hacer una cantidad exacta de decenas o centenas y corrigiendo después el resultado:

$56+17=56+(20-3)=(56+20)-3=73$.

b. Resta completando las decenas o centenas del sustraendo y corrigiendo después el resultado:

$265-199=265-(200-1)=265-200+1$.

c. Multiplicación por un número cualquiera de nueves: $9,99 \ldots$

$n \times 9.999=(n \times 10.000)-n: 84 \times 9=84 \times(10-1)=840-84=756 ; 47 \times 99=47 \times(100-1)=4700-47=4653$. 
